\title{
Factors Guarantee Competence of Laparoscopic Repair of Inguinal Hernia
}

\author{
Amr Elheny ${ }^{1}$, Khaled Mahran ${ }^{2}$, Abdel F Saleh ${ }^{3}$
}

\begin{abstract}
Aim: To evaluate the impact of age, type of hernia, size of the mesh used, and fixation of the mesh on the competence of laparoscopic repair of inguinal hernia.

Materials and methods: Randomized controlled clinical study carried out from November 2016 to July 2017 in 98 patients with inguinal hernias admitted to surgery Department of Minia University Hospital. Patients were divided into two groups randomly. Group I includes 49 patients who underwent laparoscopic transabdominal preperitoneal (TAPP) hernioplasty and group II includes 49 patients who underwent laparoscopic totally extra peritoneal (TEP) hernioplasty with and without fixation of the mesh.

Results: Operative time in group I ranges between 40 minutes and 110 minutes with mean time of about 66.85 minutes, while in group II ranges between 20 minutes and 105 minutes with mean time of about 52.65 minutes. This difference was statistically significant. Pain was $8.2 \%$ in group I and $10.2 \%$ in group II. Scrotal edema was $0 \%$ in all patients in both groups. Urinary retention was $2 \%$ in group I and $4.1 \%$ in group II. Seroma was the same (6.1\%) in both groups. Recurrence after 6-month follow-up was $2 \%$ in both groups. All recurrent cases are nonfixed.

Conclusion: There is no difference between TEP and TAPP, but TAPP technique appears to be superior to the TEP repair in patients undergoing unilateral inguinal hernia repair.

Clinical significance: The TEP approach can be offered to patients with bilateral and recurrent hernias. TEP procedure was associated with more adverse events during surgery but less postoperative pain, faster recovery of daily activities, quicker return to work, and less impairment of sensibility after 1 year.

Keywords: Inguinal hernia, Laparoscopic, Minia.

World Journal of Laparoscopic Surgery (2018): 10.5005/jp-journals-10033-1353
\end{abstract}

\section{INTRODUCTION}

Inguinal hernias comprise approximately $7 \%$ of all surgical outpatient visits. Male-to-female ratio is $8: 1$. They affect $1-3 \%$ of young children. In men, the incidence rises from 11 per 10,000 person-years, aged 16-24 years, to 200 per 10,000 person-years, aged 75 years or above. ${ }^{1}$

Conventionally, there are two types of inguinal hernia: Indirect-a protrusion through the internal inguinal ring passes along the inguinal canal through the abdominal wall, running laterally to the inferior epigastric vessels. This is the more common form accounting for $80 \%$ of inguinal hernias, especially in children. It is associated with failure of the inguinal canal to close properly after passage of the testis in utero or during the neonatal period. Directthe hernia protrudes directly through a weakness in the posterior wall of the inguinal canal, running medially to the inferior epigastric vessels. It is more common in the elderly and rare in children. ${ }^{2}$

Inguinal hernia repair is the most frequently performed operation in general surgery. The standard method for inguinal hernia repair had changed little over a hundred years until the introduction of synthetic mesh. This mesh can be placed by either using an open approach or by using a minimal access laparoscopic technique. ${ }^{3}$

Although open mesh-based tension-free repair remains the standard operation, laparoscopic hernioplasty in the hands of adequately trained surgeons produces excellent results comparable to those of open repair. ${ }^{4-6}$

The techniques of laparoscopic hernioplasty include TAPP, TEP approach, and intraperitoneal onlay mesh (IPOM)
${ }^{1-3}$ Department of General Surgery, Minia University, Minia, Egypt

Corresponding Author: Amr Elheny, Department of General Surgery, Minia University, Minia, Egypt, Phone: +20 1277340340, e-mail: amr_ elheny@yahoo.com

How to cite this article: Elheny A, Mahran K, et al. Factors Guarantee Competence of Laparoscopic Repair of Inguinal Hernia. World J Lap Surg 2018;11(3):124-127.

Source of support: Nil

Conflict of interest: None

technique. The three techniques are based on the principles of using mesh prosthesis to cover the defect of the abdominal wall from inside. ${ }^{7-9}$

The advantages over conventional surgery are primarily pain reduction, shorter length of hospital stay, and faster resumption of usual activities. In addition, many studies also show lower morbidity rates and less impairment of the immune system. ${ }^{10}$

Disadvantages of the laparoscopic technique compared with conventional surgery include the higher operating room costs, time consuming specially in the early learning curve of the surgeons, and the need for general anesthesia. ${ }^{11}$

\section{Objective}

The purpose of this study was to evaluate the impact of age, type of hernia, size of the mesh used, and fixation of the mesh on the competence of laparoscopic repair of inguinal hernia. 


\section{Materials and Methods}

This is a randomized controlled clinical study carried out from November 2016 to July 2017 in 98 patients with inguinal hernias admitted to surgery department of Minia University hospital. Informed consent was taken. All patients were operated upon laparoscopically using prolene mesh. Patients were divided into two groups randomly. Group I includes 49 patients who underwent laparoscopic TAPP hernioplasty with fixation and without fixation of the mesh, and group II includes 49 patients who underwent laparoscopic TEP hernioplasty with and without fixation of the mesh. The mesh was in a size of $7.5 \times 11 \mathrm{~cm}$; doubling of mesh had been done in some cases, others tailoring of the mesh. Tailored corner of mesh was positioned infero-medially over the Cooper's ligament and pubic bone fixed using a secure strap, while the superior border of the mesh was fixed to posterior rectus and fascia transversalis in TAPP.

Demographic and clinical data were analyzed (age, type of hernia, operating time, size of the mesh, fixation of the mesh, length of hospital stay, recurrence, intraoperative and postoperative complications). Rules for preoperative correction of general diseases and precipitating factors of hernia recurrence were followed.

Patients were hospitalized the day before surgery and underwent routine preoperative evaluation including chest X-ray, ECG, laboratory studies, and abdominal ultrasound.

Prophylactic broad spectrum antibiotic (amoxicillin + clavulanic acid) was administered at the induction of anesthesia. Some patients were operated upon under general anesthesia others under spinal anesthesia (then converted to general anesthesia due to accidental opening of the peritoneum) with Foley catheter inserted in some cases of TAPP.

The postoperative care of laparoscopic patients immediately after the completion of the surgical procedure is important and includes appropriate monitoring during the early postoperative period usually in the recovery room to ensure a smooth transition from the anesthetic. Most patients require only routine assessment of vital signs. Acutely ill patients or those with significant cardiac or pulmonary disease will require invasive monitoring in an intensive care unit. Appropriate fluids should be administered with consideration to the extent of the dissection, unless there is a specific reason to leave the Foley catheter in place they should be removed.

Pain management following laparoscopy is generally easier than following other more invasive surgical procedures. Pain is generally much less with laparoscopy, one of the primary advantages of this approach. Postoperative analgesia may consist solely of oral medication. Advanced or lengthy procedures may be accompanied by more pain than simple procedures. Diaphragmatic irritation is an important source of postoperative pain and may lead to complains of shoulder or neck discomfort. By the postoperative first day, intensity of the surgical pain generally decreases significantly and at this point, patients can be maintained on oral pain medication exclusively.

Depending on the procedure, resumption of oral intake can begin sooner than with other types of surgery. Following laparoscopic hernioplasty liquids can be provided as soon as the patient awakens from the anesthesia or shortly thereafter, patients are advanced to a normal diet and prepared for discharge. Patients can generally return to work 48 hours after a laparoscopic hernia repair if they are not required to perform heavy lifting or straining. If the patient is doing well without complications, they may resume any heavy lifting, straining, or exercise two weeks after laparoscopic hernia repair. To avoid anxiety in patients, they should be forewarned about the possibility of $\mathrm{CO}_{2}$ trapped in the scrotum, seroma formation, and discoloration of the scrotum and penis developing a few days after the operation.

Patients are discharged either on the day of operation or on the following day, others occasionally having to remain in hospital because of previous medical conditions. All repairs are reviewed in the clinic two weeks postoperatively and any early complications noted.

\section{Ethical Approval}

The title, aim, and plan of the study were discussed and approved regarding ethics of research in General Surgical Department, Minia Faculty of Medicine. Full written, informed consent was obtained from all participants. Manuscript was ethically conducted in accordance with Declaration of Helsinki.

\section{Results}

This study was conducted on 98 patients with inguinal hernia. All patients had laparoscopic surgical repair with prolene mesh. One female and 97 males suffering from inguinal hernia were included in the study with a mean age of $42.87 \pm 15.02$ years old (range $18-73$ years) in group I, $36.3 \pm 15.18$ years old (range $18-77$ years) in group II.

The side of hernia in group I was right in $28.6 \%$ of patients, left in $61.2 \%$ of them, and bilateral in $10.2 \%$, while in group II $34.7 \%$ were right, $61.2 \%$ were left, and bilateral in $4.1 \%$. The type of hernia in group I was direct in $31.5 \%$ of patients, indirect incomplete (pubonocele or funicular) in $59.2 \%$ of them, and indirect complete in $10.2 \%$, while in group II $25.5 \%$ were direct, $64.7 \%$ were indirect incomplete (pubonocele or funicular), and indirect complete in 9.8\% (Table 1).

Operative time in group I ranges between 40 and 110 minutes with mean time of about 66.85 minutes, while in group II ranges between 20 and 105 minutes with mean time of about 52.65 minutes. This difference was statistically significant (Table 2).

We used single mesh, doubled mesh, and tailoring of the mesh done in some cases. In group I, fixation of the mesh was done in $46.9 \%$ of the cases, while in group II fixation was done in $42.9 \%$ (Table 3).

Table 1: Patient's demographics

\begin{tabular}{lcll}
\hline & $\begin{array}{l}\text { Group I (TAPP) } \\
(n=49)\end{array}$ & $\begin{array}{l}\text { Group II (TEP) } \\
(n=49)\end{array}$ & p value \\
\hline Age: (years) & & & \\
$\quad$ Range & $(18-73)$ & $(18-77)$ & $0.034^{*}$ \\
$\quad$ Mean \pm SD & $42.87 \pm 15.02$ & $36.3 \pm 15.18$ & \\
Sex & & & \\
$\quad$ Male & $49(100 \%)$ & $48(98 \%)$ & 0.315 \\
$\quad$ Female & $0(0 \%)$ & $1(2 \%)$ & \\
Side of the hernia & & & \\
$\quad$ RT & $14(28.6 \%)$ & $17(34.7 \%)$ & 0.455 \\
$\quad$ LT & $30(61.2 \%)$ & $30(61.2 \%)$ & \\
$\quad$ Bilateral & $5(10.2 \%)$ & $2(4.1 \%)$ & \\
Types of hernia & & & \\
$\quad$ Direct & $17(31.5 \%)$ & $13(25.5 \%)$ & 0.793 \\
Indirect incomplete & $32(59.2 \%)$ & $33(64.7 \%)$ & \\
$\quad$ Indirect complete & $5(9.3 \%)$ & $5(9.8 \%)$ & \\
\hline
\end{tabular}

*Statistically significant 
Table 2: Operative time

\begin{tabular}{llll}
\hline & $\operatorname{TAPP}(n=49)$ & $\operatorname{TEP}(n=49)$ & p value \\
\hline Operative time: $(\min )$ & & & \\
$\quad$ Range & $(40-110)$ & $(20-105)$ & $<0.001^{*}$ \\
Mean \pm SD & $66.85 \pm 17.46$ & $52.65 \pm 16.54$ & \\
\hline
\end{tabular}

*Statistically significant

Table 3: Size and fixation of the mesh

\begin{tabular}{lrrr}
\hline & $\operatorname{TAPP}(n=49)$ & $\operatorname{TEP}(n=49)$ & pvalue \\
\hline Fixation of the mesh & & & \\
$\quad$ No & $26(53.1 \%)$ & $28(57.1 \%)$ & 0.685 \\
$\quad$ Yes & $23(46.9 \%)$ & $21(42.9 \%)$ & \\
Size of the mesh & & & \\
$\quad$ Single & $33(67.4 \%)$ & $41(83.6 \%)$ & 0.120 \\
$\quad$ Doubled & $5(10.2 \%)$ & $4(8.2 \%)$ & \\
$\quad$ Tailoring & $11(22.4 \%)$ & $4(8.2 \%)$ & \\
\hline
\end{tabular}

Table 4: Postoperative complications

\begin{tabular}{|c|c|c|c|}
\hline & $\operatorname{TAPP}(n=49)$ & $\operatorname{TEP}(n=49)$ & $p$ value \\
\hline \multicolumn{4}{|c|}{ Early postoperative complication } \\
\hline Pain & $4(8.2 \%)$ & $5(10.2 \%)$ & 0.727 \\
\hline Seroma & $3(6.1 \%)$ & $3(6.1 \%)$ & 1 \\
\hline Scrotal edema & $0(0 \%)$ & $0(0 \%)$ & - \\
\hline Urinary retention & $1(2 \%)$ & $2(4.1 \%)$ & 0.558 \\
\hline \multicolumn{4}{|c|}{ Late postoperative complication } \\
\hline Testicular pain & $1(2 \%)$ & $1(2 \%)$ & 1 \\
\hline Testicular atrophy & $0(0 \%)$ & $0(0 \%)$ & - \\
\hline Hydrocele & $0(0 \%)$ & $0(0 \%)$ & - \\
\hline Recurrence & $1(2 \%)$ & $1(2 \%)$ & 1 \\
\hline
\end{tabular}

Table 5: Duration of hospital stay

\begin{tabular}{lccc}
\hline & $\operatorname{TAPP}(n=49)$ & $\operatorname{TEP}(n=49)$ & $p$ value \\
\hline Discharge & & & \\
On the same day & $46(93.9 \%)$ & $48(98 \%)$ & 0.307 \\
On the next day & $3(6.1 \%)$ & $1(2 \%)$ & \\
\hline
\end{tabular}

Early complications include pain, seroma, scrotal edema, and urinary retention. Pain was $8.2 \%$ in group I and $10.2 \%$ in group II. Scrotal edema was $0 \%$ in all patients in both groups. Urinary retention was $2 \%$ in group I and $4.1 \%$ in group II. Seroma was the same (6.1\%) in both groups. Late complications after 6 months include testicular pain, testicular atrophy, hydrocele, and recurrence. Recurrence after 6-month follow-up was $2 \%$ in both groups. All recurrent cases are nonfixed. Testicular pain was $0 \%$ in both groups. Testicular atrophy after 6 -month follow-up was $0 \%$ clinically and by scrotal ultrasound early and late postoperative. Hydrocele was $0 \%$ in all patients (Table 4).

In group I, 93.9\% patients discharged on the same day while in group II, $98 \%$ discharged on the same day due to ileus post-TAPP (Table 5).

\section{Discussion}

In our study, we compare factors that guarantee competence of laparoscopic repair of inguinal hernia including size of the mesh, fixation of the mesh, age of the patient, and type of hernia.
Regarding size of the mesh, $7.5 \mathrm{~cm} \times 11 \mathrm{~cm}$ was mainly used; tailoring of the mesh done in some cases and doubling of the mesh done in other cases.

In group I, we used TAPP technique for inguinal hernia; fixation of the mesh was done in 27 cases, no fixation was needed in 22 cases. Two cases recurred in nonfixed mesh. In group II, we used TEP technique for inguinal hernia; fixation of the mesh was done in 28 cases, no fixation was needed in 21 cases. Two cases recurred in nonfixed mesh. All recurrent cases converted to open tension free repair.

In TAPP, regarding size of the mesh we used $7.5 \mathrm{~cm} \times 11 \mathrm{~cm}$ and only two cases recurred in nonfixed mesh (2\%), contrary to our results, Kapiris et al. found in series of 3017 cases of TAPP from two centers over seven years, the recurrence was 5\% in initial 325 cases when the mesh size was $11 \mathrm{~cm} \times 6 \mathrm{~cm}$. The mesh size was then increased to $15 \mathrm{~cm} \times 10 \mathrm{~cm}$ and this decreases the recurrence to $0.16 \%$ for the rest of the cases on follow-up. ${ }^{12}$ Sievers et al. after prospective study of 776 TAPP procedures found that the recurrence rate was 3.9\%, ${ }^{13}$ while Soltés et al. after 1058 laparoscopic hernioplasties TAPP done over a 10 years had an overall recurrence rate $0.96 \%$, zero conversion rate, and size of mesh enlarged from $7.5 \mathrm{~cm} \times 15 \mathrm{~cm}$ to $10 \mathrm{~cm} \times 15 \mathrm{~cm} .{ }^{14}$ Also, Hussain et al. had a recurrence rate of $0.18 \%$ in their study done between September 1999 and July 2009, on more than 2000 patients who underwent transabdominal preperitoneal repair of groin hernia. ${ }^{15}$

Bátorfi concluded in their study which was done between March 1994 and February 1997 on 160 TAPP that in five cases (3.1\%) early recurrences were considered to be caused by technical inexperience and/or too small prosthetic patch. ${ }^{16}$

Regarding operative time in group I ranges between (40 and 110 minutes) with mean time of about ( 66.85 minutes), contrary to our results, Soltés et al., over a period of 10 year period and 1058 laparoscopic hernioplasties done, had a mean operating time of $60 \pm 24$ minutes (30-175), with $46 \pm 19$ minutes in the last three years (last 541 patients). ${ }^{14}$ In addition, the team of Bátorfi, in 11 years (1994-2005) carried out transabdominal preperitoneal herniorrhaphies in 964 patients and had an average operation time of 112 minutes (52-195), in monolateral hernias during the learning curve and this was reduced to 57 minutes (40-125). ${ }^{16}$

Regarding length of stay in group I, $93.9 \%$ patients discharged on the same day. Wilhelm et al. investigated 249 patients underwent TAPP procedures and they found that the second day after surgery was judged to be the ideal time point for discharge by $81 \%$ of all patients whereas previously that had only been possible in $5 \%$. Accordingly, the postoperative length of stay (including the day of surgery) was significantly reduced from $4.2 \pm 0.6$ to $3.3 \pm 0.6$ days. ${ }^{17}$

Fixation of the mesh was done by secure strap in TEP 21 cases, nonfixation was done in 28 cases and in TAPP, fixation of the mesh was done in 23 cases, nonfixation was done in 26 cases; no recurrence occurs in fixed mesh. Contrary to our results, Craig Taylor et al. found that mesh fixation appears to be unnecessary in TEP repair of small hernial defects. It is associated with higher operative costs and an increased likelihood of developing chronic groin pain. The omission of mesh fixation did not increase the risk of early hernia recurrence. ${ }^{18} \mathrm{Garg}$ et al. conclude with reasonable confidence that TEP inguinal hernia repair performed without mesh fixation is safe and feasible with minimal recurrence rates. ${ }^{19}$

In addition, the patients in group II needed more pain medication immediately after the surgery than group I and urinary retention in group I $2 \%$ and in group || $4.1 \%$. Increased postoperative 
pain might lead to an increased incidence of urinary retention by increasing sympathetic tone causing urine retention. A second explanation for decreased urinary retention in patients in group II is that decreased pain leads to decreased use of postoperative analgesia. Mulroy hypothesized that increased postoperative pain might lead to an increased incidence of urinary retention by increasing sympathetic tone impeding urination. ${ }^{20}$

No incidence of hydrocele in both groups. Contrary to our results, Bátorfi in 11 years (1994-2005) and in 964 TAPP procedures found that sero-haematoma $(86=7.1 \%)$ which is the most common mild complication did not occur after the introduction of routine preperitoneal drainage. Also, Bátorfi, between March 1994 and February 1997 on 160 TAPP found 20 (12\%) cord/scrotal transient seromas-hematomas and 2 (1.2\%) hydrocele. ${ }^{16}$ However, Hussain et al. between September 1999 and July 2009 had a study on more than 2000 patients whom underwent transabdominal preperitoneal repair of groin hernia and a hematoma was reported in six patients $(0.27 \%)$, with two patients $(0.09 \%)$ needed blood transfusion whereas one patient needed re-exploration and four (0.1\%) hydroceles were confirmed. ${ }^{15}$

The patients with mesh fixation had a significantly higher incidence of seroma formation than the patients with nonfixation of the mesh. The exact reason for this could not be ascertained. A possible explanation is irritation of the peritoneum by the metallic tacks, leading to more serum formation. Also, compartmentalization of the preperitoneal space by mesh fixation may lead to a delayed resolution of physiologic serous collection in the dissected space, with later presentation of seroma. ${ }^{15}$

We found that dissection was very difficult in long-standing hernia with more than one year complain. Thus, we prefer to do incomplete dissection from the start to decrease operative time, injury to cord structure, postoperative pain, urinary retention, hospital stay, and scrotal edema. In addition, as regards the leftsided hernia, we observed that the dissection was more difficult than right-sided hernia for the right-handed surgeon.

\section{Conclusion}

There is no difference between TEP and TAPP, but TAPP technique appears to be superior to the TEP repair in patients undergoing unilateral inguinal hernia repair.

\section{Clinical Significance}

The TEP approach can be offered to patients with bilateral and recurrent hernias. TEP procedure was associated with more adverse events during surgery but less postoperative pain, faster recovery of daily activities, quicker return to work, and less impairment of sensibility after 1 year.

\section{ACKNOWLedgments}

The authors would like to offer their sincere thanks to all people who participated in the study.

\section{References}

1. Campanelli G, Canziani M, et al. Inguinal hernia: state-of-the art. Int J Surg 2008;6(Suppl 1):S26-S28. DOI: 10.1016/j.ijsu.2008.12.021.

2. van Wessem KJ, Simons MP, et al. The etiology of indirect inguinal hernias: congenital and/or acquired hernia? Hernia 2003;7(2):76-79. DOI: 10.1007/s10029-002-0108-7.

3. McCormack K, Scott NW, et al. EU Hernia Trialists collaboration. laparoscopic techniques vs open techniques for inguinal hernia repair. Cochrane Database Syst Rev 2003;1:CD001785.

4. Kuhry E, van Veen RN, et al. Open or endoscopic total extraperitoneal inguinal hernia repair? A systematic review. Surg Endosc 2007;21(2):161-166. DOI: 10.1007/s00464-006-0167-4.

5. Quilici PJ, Greaney Jr EM, et al. Laparoscopic inguinal hernia repair: optimal technical variations and results in 1700 cases. Am Surg 2000;66(9):848-852.

6. Fazzio FJ. Cost effective, reliable, laparoscopic hernia repair: a report on 500 consecutive repair. Surg Endues 2002;16(6):931-935. DOI: 10.1007/s004640080073.

7. Takata MC, Duh QY. Laparoscopic inguinal hernia repair. Surg Clin North Am 2008;88(1):157-178. DOI: 10.1016/j.suc.2007.10.005.

8. Hamza Y, Gabr E, et al. Four-arm randomized trial comparing laparoscopic and open hernia repairs. Int J Surg 2010;8(1):25-28. DOI: 10.1016/j.ijsu.2009.09.010.

9. Kumar B. Surgical options in inguinal hernia: Which is the best? J MAS 2006;4:191-200.

10. Bittner R. Laparoscopic Surgery-15 Years After Clinical Introduction. World J Surg 2006;30(7):1190-1203. DOI: 10.1007/s00268-005-0644-2.

11. Bay-Nielson M, Nilsson E, et al. Chronic pain after open mesh and sutured repair of indirect inguinal hernia in young males. Br J Surg 2004;91(10):1372-1376. DOI: 10.1002/bjs.4502.

12. Kapiris SA, Brough WA, et al. Laparoscopic transadominal preperitoneal (TAPP) hernia repair. Surg Endosco 2001;15(9):972-975. DOI: $10.1007 / \mathrm{s} 004640080090$.

13. Sievers $D$, Barkhausen $S$, et al. Laparoscopic transperitoneal inguinal hernia repair (TAPP) - complications and results of a prospective study. Langenbecks Arch Chir Suppl Kongressbd 1997;114: 1116-1118.

14. Soltés $M$, Pazinka $P$, et al. Laparoscopic hernioplasty TAPP in treatment of groin hernia-10 years experience. Rozhl Chir 2010;89(6):384-389.

15. Hussain A, Nicholls J, et al. Technical tips following more than 2000 transabdominal preperitoneal (TAPP) repair of the groin hernia. Surg Laparosc Endosc Percutan Tech 2010;20(6):384-388. DOI: 10.1097/ SLE.0b013e3182006845.

16. Bátorfi J. The treatment of inguinofemoral hernias with laparoscopic herniorraphy. Our experience of 1210 transabdominal preperitoneal (TAPP) reconstructions. Magy Seb 2005;58(6):385-397.

17. Wilhelm $\mathrm{W}$, Vassiliadis $\mathrm{N}$, et al. Optimization of perioperative management in laparoscopic hernioplasty. Anaesthesist 2008;57(9):915-925. DOI: 10.1007/s00101-008-1402-2.

18. Taylor C, Layani L, et al. Laparoscopic inguinal hernia repair without mesh fixation, early results of a large randomised clinical trial. Surg Endosc 2008;22(3):757-762. DOI: 10.1007/s00464-007-9510-7.

19. Garg $P$, Rajagopal $M$, et al. Laparoscopic total extraperitoneal inguinal hernia repair with nonfixation of the mesh for 1692 hernias. Surg Endosc 2009;23:1241-1245. DOI: 10.1007/s00464-008-0137-0.

20. Mulroy MF. Hernia surgery, anesthetic technique, and urinary retention-apples, oranges, and kumquats? Reg Anesth Pain Med 2002;27(6):587-589. DOI: 10.1097/00115550-200211000-00008. 